\title{
Formation of Poly-Si Films on Glass Substrates by Using Microwave Plasma Heating and Fabrication of TFT's on the Films
}

\section{Hiroki Nakaie1, Tetsuji Arai', Keisuke Arimoto', Junji Yamanaka1, Kiyokazu Nakagawa1, Kazuki Kamimura', Toshiyuki Takamatsu ${ }^{2}$}

${ }^{1}$ Interdisciplinary Graduate School of Medicine and Engineering, University of Yamanashi, Kofu, Japan

${ }^{2}$ SST Inc., Yachiyo, Japan

Email:g15df003@yamanashi.ac.jp

How to cite this paper: Nakaie, H., Arai, T., Arimoto, K., Yamanaka, J., Nakagawa, K., Kamimura, K. and Takamatsu, T. (2018) Formation of Poly-Si Films on Glass Substrates by Using Microwave Plasma Heating and Fabrication of TFT's on the Films. Journal of Materials Science and Chemical Engineering, 6, 19-24.

https://doi.org/10.4236/msce.2018.61003

Received: August 11, 2017

Accepted: January 1, 2018

Published: January 4, 2018

\begin{abstract}
We have developed an apparatus for producing high-density hydrogen plasma. The atomic hydrogen density was $3.0 \times 10^{21} \mathrm{~m}^{-3}$ at a pressure of $30 \mathrm{~Pa}$, a microwave power of $1000 \mathrm{~W}$, and a hydrogen gas flow rate of $5 \mathrm{sccm}$. We confirmed that the temperatures of tungsten films increased to above $1000^{\circ} \mathrm{C}$ within $5 \mathrm{~s}$ when they were exposed to hydrogen plasma formed using the apparatus. We applied this phenomenon to the selective heat treatment of tungsten films deposited on amorphous silicon films on glass substrates and formed polycrystalline silicon films. To utilize this method, we can perform the crystalline process only on device regions. TFTs were fabricated on the polycrystalline silicon films and the electron mobilities of $60 \mathrm{~cm}^{2} / \mathrm{Vs}$ were obtained.
\end{abstract}

\section{Keywords}

Microwave Plasma Heating, Poly-Si, Thin Film Transistor

\section{Introduction}

Polycrystalline silicon (poly-Si) thin film transistors (TFT's) are used for driving elements of flat panel displays (FPDs) such as liquid crystal display, organic light emitting diode display [1] [2]. Poly-Si films on a glass substrate obtained by solid phase crystallization (SPC) has been investigated [3] [4]. In general, The SPC method requires heating treatment for a very long time in order to improve crystallinity. The process temperature reaches approximately $800^{\circ} \mathrm{C}$. Therefore, we must consider that heat treatment causes the degradation of TFTs reliability. 
To reduce the effects of heat treatment, laser annealing was used to obtain poly-Si layers. However, laser annealing requires a complex and expensive apparatus. To overcome these problems, we have proposed a simple heating method, in which transition metals can be heated selectively by exposing them to hydrogen plasma. Mozetic and coworkers reported that the temperature of nickel was increased by the irradiation of hydrogen atoms and reached approximately $300^{\circ} \mathrm{C}$, and from this phenomenon they estimated the density of hydrogen atoms in hydrogen plasma. This phenomenon is attributed to the release of binding energy by the recombination of hydrogen atoms into molecules on nickel surfaces, and as a result, the nickel is heated. We have developed a heating apparatus to form high-density hydrogen plasma, in which transition metals such as nickel and tungsten can be heated to approximately $1000^{\circ} \mathrm{C}$ [5].

In this paper, we report that amorphous silicon (a-Si) layers were crystallized by plasma heating of tungsten regions deposited on a-Si/glass substrates. We also present a fabrication process poly-Si TFT's and measured electron mobilities.

\section{Experimental Methods}

\subsection{Crystallization}

$50 \mathrm{~nm}$-thick a-Si layers were deposited on quartz glass substrate by molecular beam epitaxy (MBE) at room temperature. Then, $60 \mathrm{~nm}$-thick nickel films deposition by thermal evaporation and successive $150 \mathrm{~nm}$-thick tungsten films deposition by sputtering at room temperature were performed on regions which were patterned using lift-off process (Structure A). We also prepared samples without nickel films (Structure B). The a-Si films were then crystallized in the solid phase using microwave plasma heating. Input microwave power is $1000 \mathrm{~W}$, hydrogen gas flow rate is $5 \mathrm{sccm}$, treatment time is $60 \mathrm{~s}$, and pressure is $30 \mathrm{~Pa}$.

\subsection{Poly-Silicon TFT Fabrication}

For device fabrication, $100 \mathrm{~nm}$-thick a-Si layers were deposited on quartz glass substrate by $\mathrm{MBE}$ at room temperature. Then, samples with structure A and B were prepared. tungsten and nickel were patterned only inside the source and drain regions, and the pattern sizes are smaller than source and drain regions. The microwave plasma heating method was used to perform crystallization. Input microwave power is $1000 \mathrm{~W}$, hydrogen gas flow rate is $5 \mathrm{sccm}$, treatment time is $60 \mathrm{~s}$, and pressure is $30 \mathrm{~Pa}$. The sample with the structure B was heated for $120 \mathrm{~s}$ in addition to heating time of $60 \mathrm{~s}$.

Then, source and drain regions, which were larger than the metal patterned regions, were implanted with arsenic atoms at a dose of $1.0 \times 10^{15} \mathrm{~cm}^{2}$ and an energy of $50 \mathrm{KeV}$. Gate $\mathrm{SiO}_{2}$ layers $150 \mathrm{~nm}$ thick were deposited by plasma enhanced chemical vapor deposition (PECVD) using tetraethyl orthosilicate (TEOS) at temperature of $450^{\circ} \mathrm{C}$. Then, the contact holes were patterned by wet etching using hydrofluoric acid. Finally, aluminum films deposited by thermal evaporation and patterned by wet etching to form the contact pad. Sintering 
temperature was $350^{\circ} \mathrm{C}$ in mixture of nitrogen and hydrogen gases.

\section{Results and Discussion}

\subsection{Crystallization}

To investigate the crystallinity of Si layers, we used optical microscope and Raman scattering spectroscope. The optical micrograph of the sample with the structure B after heat treatment is shown in Figure 1. The color of a-Si film was red and that of crystallized silicon film was yellow. The poly-Si aria was obtained near tungsten region. The length of the poly-Si aria was about $2 \mathrm{~mm}$.

The crystallinity of the sample with the structure B after plasma heating was investigated by microscope Raman spectroscopy. Figure 2(a) shows the spectrum of the yellow aria in Figure 1. The spectrum has a Si-Si TO-LO phonon band centered at $520 \mathrm{~cm}^{-1}$, showing the presence of Si crystalline phase [6]. Figure 2(b) shows the spectrum of the red aria in Figure 1. The spectrum has broad peak centered at $480 \mathrm{~cm}^{-1}$, it's characteristic of a-Si.

The optical micrograph of the sample with the structure A after plasma heating was shown in Figure 3. Even though heat treatment was performed for the same period of time as the sample with structure $B$, whole area of the sample with structure A $(2 \mathrm{~mm} \times 15 \mathrm{~mm})$ was crystallized.

The length of polycrystallized region in Figure 3. was larger than that in Figure 1. It is thought to be attributed to metal induced lateral crystallization

\begin{tabular}{|l|l|}
\hline$W$ & \\
\hline Poly-Si a-Si: \\
\hline & glass substrate \\
\hline
\end{tabular}

(a)

W Poly-Si a-Si $\frac{}{2 \mathrm{~mm}}$

(b)

Figure 1. (a) Cross-sectional structure and (b) plan view optical image of sample with structure B after plasma heating for $60 \mathrm{~s}$.

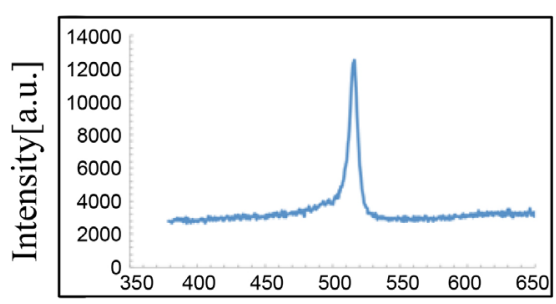

Raman shift $\left[\mathrm{cm}^{-1}\right]$

(a)

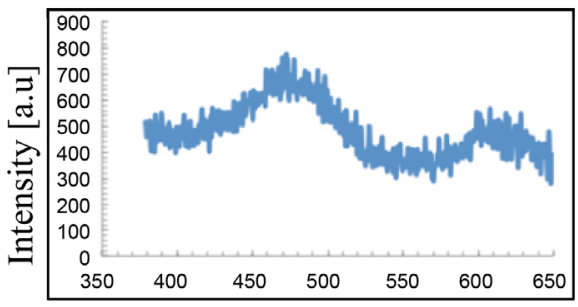

Raman shift $\left[\mathrm{cm}^{-1}\right]$

(b)

Figure 2. Microscope Raman spectra of sample with structure A after plasma heating for $60 \mathrm{~s}$ obtained at (a) red area and (b) yellow area in Figure 1. 
(MILC) by nickel [7] [8] [9].

\subsection{Poly-Silicon TFT Fabrication}

Figure 4 and Figure 5 show $I_{d}-V_{d}$ characteristic of the sample with structure $B$ (without $\mathrm{Ni}$ ) which was heated $60 \mathrm{~s}$ and $120 \mathrm{~s}$ respectively. Device dimensions are $100 \mu \mathrm{m}$ of a channel length and $150 \mu \mathrm{m}$ of a channel width. The gate voltage was changed from $0 \mathrm{~V}$ to $40 \mathrm{~V}$ with $5 \mathrm{~V}$ increment.

Drain current of the $120 \mathrm{~s}$ heat treated sample with structure B was twice larger than those of the $60 \mathrm{~s}$ heat treated samples.

Effective electron mobilities were derived using the split $\mathrm{C}-\mathrm{V}$ method. The mobilities of the $60 \mathrm{~s}$ and $120 \mathrm{~s}$ heat treated samples with structure $\mathrm{B}$ are 30 $\mathrm{cm}^{2} / \mathrm{Vs}$ and $60 \mathrm{~cm}^{2} / \mathrm{Vs}$, respectively. By elongating the plasma heating time, it was thought to obtain higher electron mobility.

Figure 6 shows $I_{d}-V_{d}$ characteristic of the $60 \mathrm{~s}$ heat treated sample with structure A (with $\mathrm{Ni}$ ). The electron mobility is $55 \mathrm{~cm}^{2} / \mathrm{Vs}$. Despite the same plasma heating time, the mobility of sample $A$ is about twice as high as that of the sample with structure $B$.

\begin{tabular}{|c|}
\hline $\mathrm{W}$ \\
\hline $\mathrm{Ni}$ \\
\hline \multicolumn{2}{|c|}{ Poly-Si } \\
\hline glass substrate \\
\hline
\end{tabular}

(a)

$\begin{array}{lll}\mathrm{W} / \mathrm{Ni} & \text { Poly-Si } & \\ & \end{array}$

(b)

Figure 3. (a) Cross-sectional structure and (b) plan view optical image of sample with structure A after plasma heating for $60 \mathrm{~s}$.

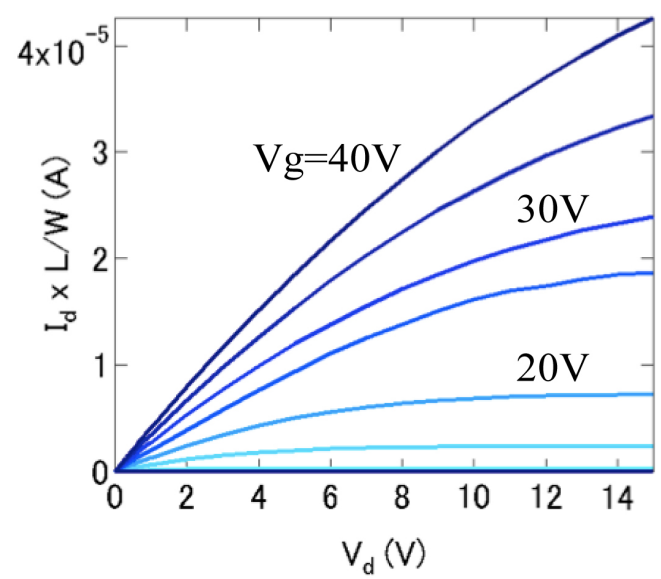

Figure 4. $I_{d}-V_{d}$ characteristics of the sample with structure B after annealing for $60 \mathrm{~s}$. 


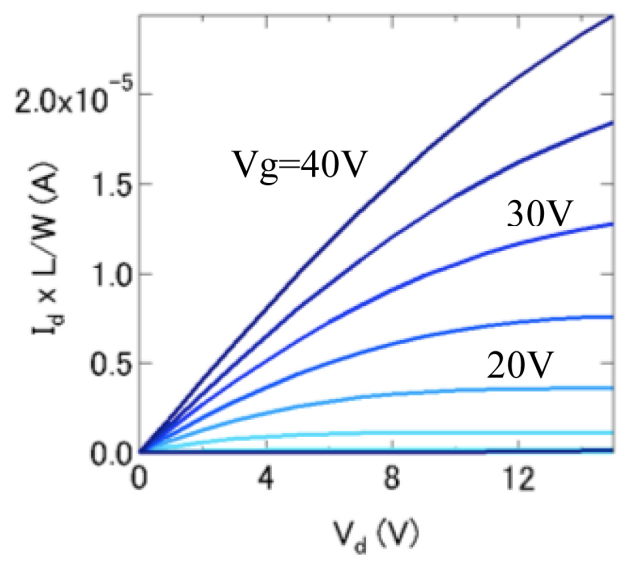

Figure 5. $I_{d}-V_{d}$ characteristics of the sample with structure B after annealing for $120 \mathrm{~s}$.

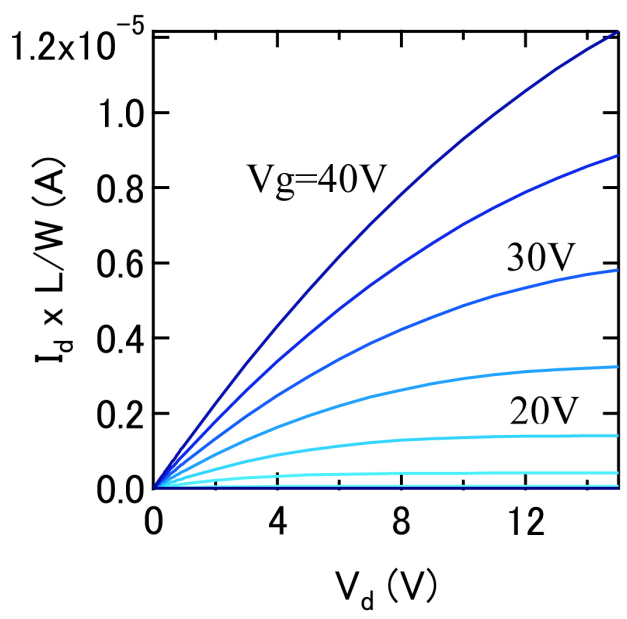

Figure 6. $I_{d}-V_{d}$ characteristics of the sample with structure A after annealing for $60 \mathrm{~s}$.

\section{Summary}

We have developed an apparatus for producing high-density hydrogen plasma. When transition metals were exposed to hydrogen plasma, the temperatures increased to above $800^{\circ} \mathrm{C}$. We applied this phenomenon to the selective heat treatment of tungsten films deposited on amorphous silicon films on glass substrates and formed polycrystalline silicon films. The characteristic of this crystallization technique is to be able to crystallize a-Si films not only just beneath the transition metal films but also laterally in the order of several millimeters. The TFTs were fabricated on poly-Si films formed by the selective plasma heating method and the electron mobilities of $60 \mathrm{~cm}^{2} / V s$ were obtained. These values are almost the same values as those of the TFT's for driving elements of FPDs.

Current main method for crystallization is laser annealing one, in which multiple scanning is needed for this purpose. Furthermore, laser annealing is unable to heat selectively. Our developed method realizes selective and rapid heating process. 


\section{References}

[1] Stewart, M., Howell, R.S., Pires, L. and Hatalis, M.K. (2001) Polysilicon TFT Technology for Active Matrix OLED Displays. IEEE Transactions on Electron Devices, 48, 845-851. https://doi.org/10.1109/16.918227

[2] Hara, A., Takeuchi, F. and Sasaki, N. (2002) Mobility Enhancement Limit of Excimer-Laser-Crystallized Polycrystalline Silicon Thin Film Transistors. Journal of Applied Physics, 91, 708-714. https://doi.org/10.1063/1.1420766

[3] Matsuyama, T., Terada, N., Baba, T., Sawada, T., Tsuge, S., Wakisaka, K. and Tsuda, S. (1996) High-Quality Polycrystalline Silicon Thin Film Prepared by a Solid Phase Crystallization Method. Journal of Non-Crystalline Solids, 198, 940-944. https://doi.org/10.1016/0022-3093(96)00091-9

[4] Huang, G., Xi, Z. and Yang, D. (2006) Crystallization of Amorphous Silicon Thin Films: The Effect of Rapid Thermal Processing Pretreatment. Vacuum, 80, 415-420. https://doi.org/10.1016/j.vacuum.2005.07.006

[5] Arai, T., Nakaie, H., Kamimura, K., Nakamura, H., Ariizumi, S., Ashizawa, S., Arimoto, K., Yamanaka, J., Sato, T., Nakagawa, K. and Takamatsu, T. (2016) Selective Heating of Transition Metal Usings Hydrogen Plasma and Its Application to Formation of Nickel Silicide Electrodes for Silicon Ultralarge-Scale Integration Devices. Journal of Materials Science and Chemical Engineering, 4, 29.

[6] Iqbal, Z. and Veprek, S. (1982) Raman Scattering from Hydrogenated Microcrystalline and Amorphous Silicon. Journal of Physics C: Solid State Physics, 15, 377.

[7] Kim, Y. S., Kim, M.S. and Joo, S.K. (2007) Effect of Amorphous Silicon Shape on Its Metal-Induced Lateral Crystallization Rate. Thin Solid Films, 515, 3387-3390. https://doi.org/10.1016/j.tsf.2006.09.054

[8] Jin, Z., Moulding, K., Kwok, H.S. and Wong, M. (1999) The Effects of Extended Heat Treatment on Ni Induced Lateral Crystallization of Amorphous Silicon Thin Films. IEEE Transactions on Electron Devices, 46, 78-82.

https://doi.org/10.1109/16.737444

[9] Dimova-Malinovska, D., Grigorov, V., Nikolaeva-Dimitrova, M., Angelov, O. and Peev, N. (2006) Investigation of Structural Properties of Poly-Si Thin Films Obtained by Aluminium Induced Crystallization in Different Atmospheres. Thin Solid Films, 501, 358-361. https://doi.org/10.1016/j.tsf.2005.07.157 